\title{
Improvement of the Dissolution Rate of Nitrendipine Using a New Pulse Combustion Drying Method
}

\author{
Liang WANG, ${ }^{a}$ Fu-De CuI, ${ }^{a}$ and Hisakazu SunADA $*, b$ \\ ${ }^{a}$ Department of Pharmaceutics, Shenyang Pharmaceutical University; No. 103 Wenhua Road, Shenyang 110016, P. R. \\ China: and ${ }^{b}$ Faculty of Pharmacy, Meijo University; 150 Yagotoyama, Tempaku-ku, Nagoya 468-8503, Japan. \\ Received March 27, 2006; accepted April 3, 2007
}

Solid dispersions (SDs) of nitrendipine (NTD), a poorly water-soluble drug, were prepared with the Hypulcon pulse combustion dryer system, and the physicochemical properties of particles were investigated and compared with those of particles prepared with a spray dryer. The SD particles prepared with Hypulcon using Aerosil and Tween 80 as carriers showed improved properties over those prepared with a conventional spray dryer, such as smaller particle size, tighter particle size distribution, and no agglomeration. Powder X-ray diffraction and differential scanning calorimetry evaluation showed that the drug in the NTD-Aerosil SD prepared with $5 \%(\mathrm{v} / \mathrm{v})$ Tween 80 solution was dispersed in an amorphous state. Fourier transformation IR spectroscopy indicated the presence of hydrogen bonds between NTD and Aerosil. Aerosil had greater ability to improve the dissolution of NTD than Sylysia and other polymers. The highest drug supersaturation concentration was maintained continuously during the dissolution test of the NTD-Aerosil SD prepared with $5 \%$ (v/v) Tween 80 solution using Hypulcon. The good hydrophilicity and dispersibility of Aerosil, solubilization of Tween 80, and actions of shock waves and ultrasonic waves might account for the amorphization of NTD and improved dissolution rate of SDs. Pulse combustion drying with low drying costs and high thermal efficiency is a promising method for the preparation of SD particles with improved properties without using organic solvent.

Key words pulse combustion dryer system; solid dispersion; poorly water-soluble drug; dissolution; spray dryer; silica particle

The solid dispersion (SD) method is commonly employed to improve the dissolution properties of poorly water-soluble drugs. ${ }^{1-3)}$ However, the preparation of a SD requires a large amount of organic solvent to dissolve the drug and a watersoluble polymer, causing environmental pollution and toxicity due to the residual solvent. ${ }^{4)}$ Previously, we reported that SDs of a poorly water-soluble drug, nitrendipine (NTD), were prepared using the twin screw extruder method with HPMCP and Carbopol as carriers, and NTD-Carbopol SDs improved the dissolution of NTD. ${ }^{5)}$ We also demonstrated that the dissolution of NTD was improved by SDs prepared with the melt-mixing method using non porous silica particles (Aerosil 200, 300, 380) and porous silica particles (Sylysia 350,730) as carriers and the rate of dissolution from NTD-Aerosil 200 SD was the highest. ${ }^{6,7)}$ However, the high supersaturation concentration of the drug obtained after about 10 min was not maintained during the course of the dissolution test.

The pulse combustion dryer system is a relatively new apparatus for drying which generates powerful shock waves via its pulse engine, effectively breaking up a solid liquid. ${ }^{8)}$ Figure 1 illustrates one such system, the Hypulcon (Pultech Corporation, Kobe, Japan), and its pulse engine. Combustion air and gaseous fuel are drawn into the pulse combustion chamber and form a fuel-air mixture, which is ignited by a pilot and explodes, creating hot, high-pressure gases. Most of the hot gases form shock waves and rush down the tailpipe toward the atomizer, as shown in Fig. 1B. Then, more fuel and air enters and explodes again due to the hot gases left in the tailpipe. This combustion cycle repeats itself at a frequency of about 50 to $1000 \mathrm{~Hz}$ to produce consecutive high-temperature shock waves. Next, the feed material is sprayed by the atomizer into high-speed combustor exhaust gases produced by the pulse engine and is dried by the actions of shock waves, ultrasonic waves (greater than $155 \mathrm{~dB}$ ), gas flow, and gas temperature $\left(200-300^{\circ} \mathrm{C}\right)$ in the drying chamber. Finally, the dried powders are retrieved using collection equipment, such as a cyclone and bag filter.

Spray drying is well known for its ability to produce a free-flowing powder and is also used for the preparation of SD particles. ${ }^{910)}$ However, the feed material is atomized through a spray nozzle at high pressure with high mechanical shear forces, the atomization and application of heat are separate functions in space and time, and the heat transfer rate is lower and less turbulent, leading to longer drying times and higher maintenance costs such as gas and electrical inputs.

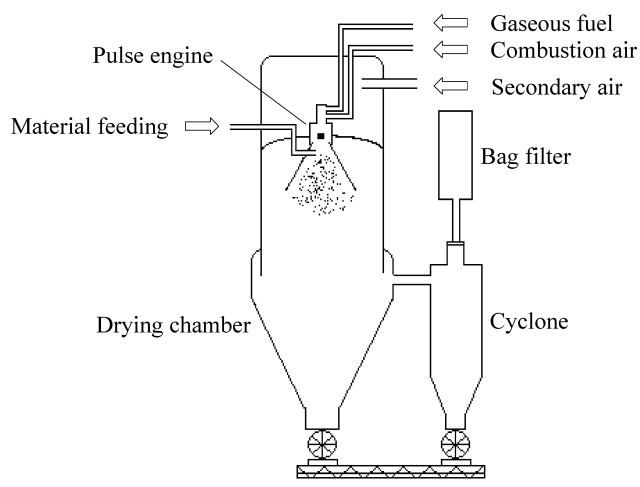

(A)

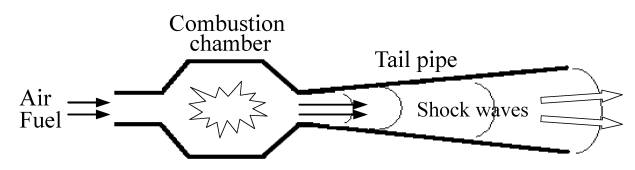

(B)

Fig. 1. Scheme of Hypulcon (A) and Pulse Engine (B) 
The pulse combustion dryer system can offer important advantages over spray drying since pulse combustion drying is accomplished by the application of not only heat for evaporation, but also the mechanical action of gas dynamic atomization, providing an environment of extreme turbulence that promotes very high rates of heat transfer and dehydration through compression and contraction. Low-temperature drying below $80^{\circ} \mathrm{C}$, instantaneous drying within $1 / 100$ th of a second, and high thermal efficiency greater than $80 \%$ are achieved. Another feature is that after drying the particles do not easily coagulate. Powders are produced with higher quality such as superior particle surface characteristics, uniform mean particle size, and tight particle size distribution. Users can also realize dramatically lower maintenance and utility costs. ${ }^{11,12)}$

A pulse combustion dryer system has been reported for drying inorganic (e.g., metal oxide, calcium carbonate), food and agricultural products, and heat-sensitive biomaterials such as antibiotics, vitamins, biopesticides, etc. ${ }^{11)}$ However, there are no reports that Hypulcon has been used for the preparation of SD. Hence, in the present study, we utilized this unique dryer system to prepare SD particles of NTD with various carriers to improve its dissolution rate and compared their physicochemical properties with those prepared with a conventional spray dryer.

\section{Experimental}

Materials NTD used as a test drug was obtained from Nanjing Pharmaceutical Factory (China). As carriers, fumed $\mathrm{SiO}_{2}$ powder Aerosil 200FAD (Nippon Aerosil, Japan), porous silica particles Sylysia 350 (Fuji Silysia Chemical, Japan), and Kollidon 30 (PVP K30, BASF AG, Ludwigshafen, Germany) were used. Polyvinyl Alcohol (PVA 500) and Tween 80 were obtained from Wako Pure Chemical Industries Ltd., Japan. All other chemicals were of analytical or HPLC grade. All experiments were carried out under reduced light to prevent the degradation of NTD.

Preparation of SD NTD and the carriers were mixed by hand in a polyethylene bag for $10 \mathrm{~min}$ with a weight ratio of $1: 5$ to obtain physical mixtures (PMs). PMs were added to water and Tween 80 solutions of different concentrations to obtain suspensions (Table 1). Then the suspensions were dried with Hypulcon or a spray dryer (SD-1000, Tokyo Rikakikai, Japan). The particles collected were stored at $40{ }^{\circ} \mathrm{C}$ in a glass desiccator with blue silica gel for $1 \mathrm{~d}$ before the testing of their properties.

Samples were dried with Hypulcon under the following operating conditions: drying chamber temperature, $60{ }^{\circ} \mathrm{C}$; secondary air fan, $60 \%$; and material feeding pump, automatic (about $8-13 \mathrm{ml} / \mathrm{min}$ ). Hypulcon is an environmentally friendly system and its pulse engine produces minimal environmental pollution with little $\mathrm{NO}_{\mathrm{x}}(20 \mathrm{ppm})$ owing to complete combustion. The components of the exhaust gas are $\mathrm{NO}_{\mathrm{x}}, \mathrm{O}_{2}$, and $\mathrm{CO}$, and their concen- trations in the exhaust gas are shown in Fig. 2. The NTD-Aerosil SD prepared with Hypulcon was analyzed using a Shodex HPLC system (Showa Denko, Tokyo, Japan), and its chromatogram was found to be similar to that of NTD-Aerosil PM (Fig. 3), indicating that $\mathrm{NO}_{\mathrm{x}}$ had no effect on the stability of the drug.

The operating conditions for the spray dryer were: inlet temperature, $135^{\circ} \mathrm{C}$; outlet temperature, $75^{\circ} \mathrm{C}$; drying air flow rate, $0.6 \mathrm{ml} / \mathrm{min}$; atomizing air pressure, $50 \mathrm{kPa}$; and sample feed rate, $10 \mathrm{ml} / \mathrm{min}$.

Confirmation of Particle Morphology The morphology of the particles was observed with a scanning electron microscope (SEM, JEOL type SM-T20).

Powder X-Ray Diffraction (PXRD) The powder X-ray diffraction analysis was performed with a linear X-ray diffraction system (RAD-2VC, Rigaku Denki Co., Ltd.) in which $\mathrm{CuK} \alpha$ rays $(40 \mathrm{kV}, 20 \mathrm{~mA})$ were used as $\mathrm{X}$-rays. The degree of diffraction was measured at $5^{\circ} / \mathrm{min}$ every $0.01^{\circ}$ between 5 and $45^{\circ}(2 \theta)$.

Table 1. Formulation of Suspensions

\begin{tabular}{|c|c|c|c|c|}
\hline Sample & $\begin{array}{c}\text { NTD } \\
(\mathrm{g})\end{array}$ & $\begin{array}{c}\text { Carrier } \\
\text { (g) }\end{array}$ & $\begin{array}{l}\text { Tween } 80 \\
(\mathrm{ml})\end{array}$ & $\begin{array}{l}\mathrm{H}_{2} \mathrm{O} \\
(\mathrm{ml})\end{array}$ \\
\hline NTD (pulse) & 10 & - & - & 500 \\
\hline Aerosil (1\% Tween 80$)$ & - & 10 & 5 & 500 \\
\hline NTD : Aerosil $=1: 5$ & 2 & 10 & - & 500 \\
\hline NTD : Sylysia $=1: 5$ & 2 & 10 & - & 500 \\
\hline NTD : PVP K30=1:5 & 2 & 10 & - & 500 \\
\hline NTD $:$ PVA $=1: 5$ & 2 & 10 & - & 500 \\
\hline NTD : Aerosil=1: 5 (1\% Tween 80$)$ & 2 & 10 & 5 & 500 \\
\hline NTD : Aerosil= $1: 5(3 \%$ Tween 80$)$ & 2 & 10 & 15 & 500 \\
\hline NTD : Aerosil $=1: 5(5 \%$ Tween 80$)$ & 2 & 10 & 25 & 500 \\
\hline NTD : Sylysia=1 : 5 (5\% Tween 80$)$ & 2 & 10 & 25 & 500 \\
\hline
\end{tabular}

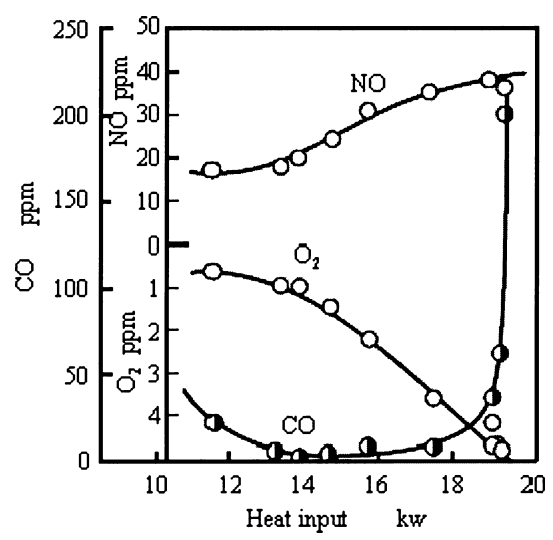

Fig. 2. Concentration of $\mathrm{NO}_{\mathrm{x}}, \mathrm{O}_{2}$, and $\mathrm{CO}$ in the Exhaust Gas The graph was obtained from the catalog of Hypulcon.

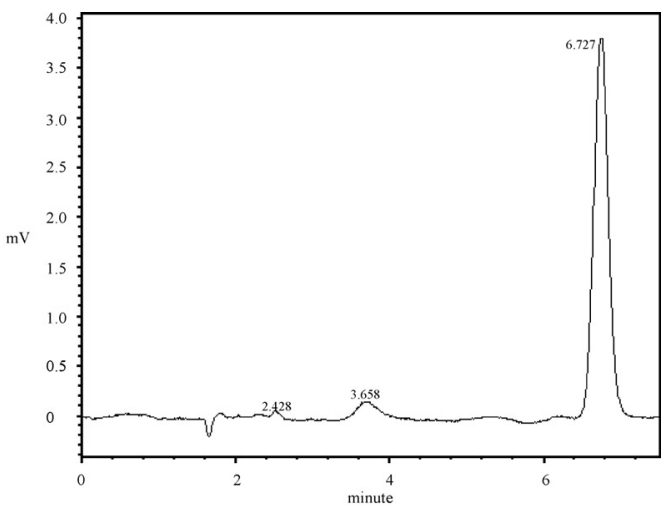

(B)

Fig. 3. HPLC Chromatograms of NTD before and after Treatment with Hypulcon

(A) NTD-Aerosil PM; (B) SD (NTD : Aerosil=1:5). 
Differential Scanning Calorimetry The differential scanning calorimetry analysis was carried out on a differential scanning calorimeter (DSC 60, Shimadzu Co., Japan). All samples were measured by placing $4-10 \mathrm{mg}$ of the powder into an aluminum pan for analysis. The thermograms were obtained by heating the samples at a rate of $10^{\circ} \mathrm{C} / \mathrm{min}$ from 30 to $180^{\circ} \mathrm{C}$ in atmospheric air. Plots of heat flow versus temperature were recorded.

Fourier Transformation IR Spectroscopy Drug-carrier interactions in the SDs were determined based on IR spectra measured with a Fourier transformation IR spectrometer (Jasco FT/IR-4100) using the KBr method in the $4000-400 \mathrm{~cm}^{-1}$ region at a $4 \mathrm{~cm}^{-1}$ resolution at 64 scans per spectrum.

Dissolution Test The amount of drug released from the samples was determined using a dissolution test apparatus (NTR-3000, Toyama Sangyo Co., Ltd.) according to dissolution test method 2 (paddle method) described in the Japanese Pharmacopoeia (JP) XIX. The paddles were rotated at $100 \mathrm{rpm}$. The samples (equivalent to $40 \mathrm{mg}$ of NTD) were added to the dissolution medium $[900 \mathrm{ml}$ of water or $0.05 \%(\mathrm{v} / \mathrm{v})$ Tween 80 solution adjusted to $37 \pm 0.1^{\circ} \mathrm{C}$ ]. Test fluids (about $5 \mathrm{ml}$ ) were withdrawn at predetermined time intervals from each vessel through a glass filter, and then filtered through a $0.2 \mu \mathrm{m}$ membrane filter (DISMIC-13CP, Toyo Filter Paper Co., Ltd.). After adding $1.4 \mathrm{ml}$ of methanol to $0.6 \mathrm{ml}$ of filtrate, the concentration of NTD was measured using the Shodex HPLC system (Showa Denko, Tokyo). The same volume of fresh medium was replaced and a correction for the cumulative dilution was calculated. The amount of NTD dissolved for each sample $(n=3)$ was plotted versus time. The HPLC conditions were: reverse-phase column, Inertsil ODS-3 $(5 \mu \mathrm{m}, 4.6 \mathrm{~mm} \phi \times 150 \mathrm{~mm}$, GL Science Inc., Tokyo, Japan) at $35^{\circ} \mathrm{C}$; mobile phase, methanol : water $=7: 3(\mathrm{v} / \mathrm{v})$ mixture; flow rate, $1.0 \mathrm{ml} / \mathrm{min}$; and detection wavelength, $236 \mathrm{~nm}$.

Solubility The solubility of NTD in water and Tween 80 solutions was determined using the dissolution test apparatus. The paddles were rotated at $100 \mathrm{rpm}$. About $2 \mathrm{~g}$ of NTD was added to the test solution $[900 \mathrm{ml}$ of water or $1 \%, 3 \%$, and $5 \%(\mathrm{v} / \mathrm{v})$ Tween 80 solutions adjusted to $\left.37 \pm 0.1^{\circ} \mathrm{C}\right]$. Tes fluids (about $5 \mathrm{ml}$ ) were withdrawn from each vessel after $48 \mathrm{~h}$ and filtered through the $0.2 \mu \mathrm{m}$ membrane filter. After the filtrate was diluted with methanol, the concentration of NTD was measured using the Shodex HPLC system.

Surface Tension The capillary rise method was used to measure the surface tension of Tween 80 solutions. When the lower end of the capillary tube is placed in a liquid, surface tension pulls the liquid column up. Then the surface tension of the liquid can be obtained using Eq. 1.

$$
\gamma=\frac{r h d g}{2 \cos \theta}
$$

where $\gamma$ is the surface tension; $r$ is the radius of the tube; $h$ is the height of the liquid column; $d$ is the density of the liquid; $g$ is acceleration due to gravity; and $\theta$ is contact angle. Water at $30^{\circ} \mathrm{C}$ has a surface tension $\left(\gamma_{\mathrm{w}}\right)$ of $71.15 \mathrm{dyn} / \mathrm{cm}$. Accordingly, the surface tension of Tween 80 solutions was calculated using Eq. 2.

$$
\gamma=\gamma_{\mathrm{w}} \frac{h}{h_{\mathrm{w}}}
$$

Stability Test The sample particles were stored in the desiccators with $75 \% \mathrm{RH}$ (saturated solution with $\mathrm{NaCl}$ ) at $40^{\circ} \mathrm{C}$ for 1 month. The crystallinity of NTD in the SD particles was measured with the powder X-ray diffraction method.

\section{Results and Discussion}

Solubility of NTD in Tween 80 Solutions The solubility of NTD in Tween 80 solutions is shown in Table 2. NTD has low solubility (about $2 \mu \mathrm{g} / \mathrm{ml}$ ) in water, which significantly increased in Tween 80 solutions. According to the calculation, about 62,178 , and $298 \mathrm{mg}$ of NTD were dissolved in $500 \mathrm{ml}$ of $1 \%, 3 \%$, and $5 \%(\mathrm{v} / \mathrm{v})$ Tween 80 solutions, respectively. It was confirmed that most NTD was not dissolved in the suspensions.

Changes in Crystallinity Powder X-ray diffraction patterns of NTD-Aerosil systems prepared with the pulse combustion dryer system are shown in Fig. 4. The original NTD drug powder showed numerous distinctive peaks (Fig. 4A)
Table 2. Solubility of NTD in Tween 80 Solutions

\begin{tabular}{lc}
\hline \hline \multicolumn{1}{c}{ Sample } & Solubility $(\mu \mathrm{g} / \mathrm{ml})$ \\
\hline $\mathrm{H}_{2} \mathrm{O}$ & 2.20 \\
1\% Tween 80 & 124.43 \\
3\% Tween 80 & 356.43 \\
5\% Tween 80 & 596.59 \\
\hline
\end{tabular}

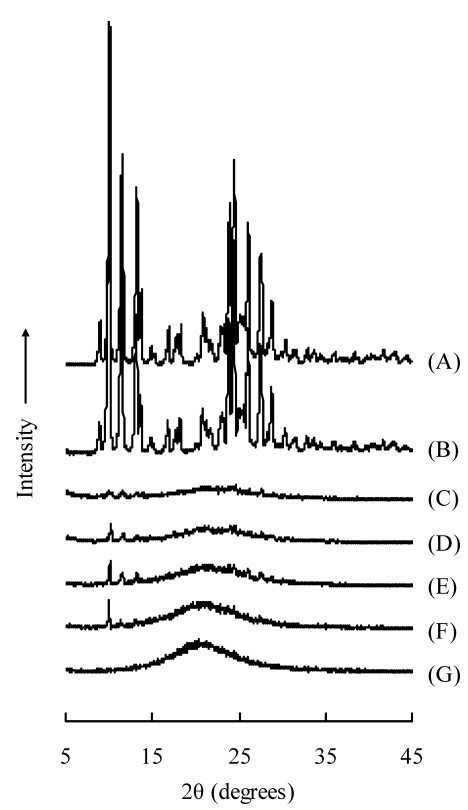

Fig. 4. Powder X-Ray Diffraction Patterns of NTD-Aerosil Systems Prepared with Hypulcon

(A) original NTD; (B) NTD (pulse); (C) PM; (D) SD without Tween 80; (E) SD (1\% Tween 80); (F) SD (3\% Tween 80); (G) SD (5\% Tween 80$)$.

that indicated high crystallinity. The NTD powder dried with Hypulcon (Fig. 4B) showed all the characteristic diffraction peaks of crystalline NTD with no decrease in their intensity, indicating that there was no change in crystallinity after the powder was dried with Hypulcon. In the NTD-Aerosil SD prepared without Tween 80 (Fig. 4D) and the SDs prepared with $1 \%(\mathrm{v} / \mathrm{v})$ and $3 \%(\mathrm{v} / \mathrm{v})$ Tween 80 solutions (Figs. 4E, F), the distinctive diffraction peaks of NTD persisted with a marked decrease in their intensity compared with the original NTD crystals. However, the NTD-Aerosil SD prepared with $5 \%(\mathrm{v} / \mathrm{v})$ Tween 80 solution (Fig. 4G) exhibited a complete halo pattern with no diffraction peaks, indicating that NTD in the SD was transformed to an amorphous state.

The DSC thermograms of NTD-Aerosil SD particles prepared with the pulse combustion dryer system (Fig. 5) showed the same results as the powder X-ray diffraction patterns. The NTD-Aerosil SD prepared with 5\% (v/v) Tween 80 solution (Fig. 5G) exhibited broad curves with no endothermic peak corresponding to the melting of pure crystalline NTD. It was considered that the crystallinity of NTD decreased and SD was formed when the concentration of Tween 80 solution increased.

The other samples prepared with Sylysia, PVP K30, and PVA as carriers exhibited the same powder X-ray diffraction patterns and DSC thermograms as the NTD-Aerosil SD prepared without Tween 80 (data not shown).

Confirmation of Interactions in the FT-IR Spectrum 


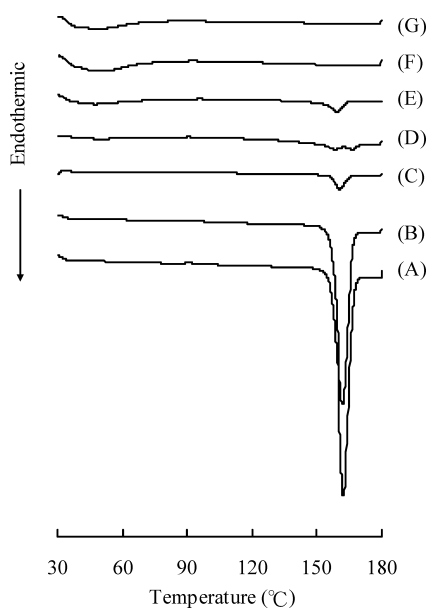

Fig. 5. Differential Scanning Calorimetry Curves of NTD-Aerosil Systems Prepared with Hypulcon

(A) original NTD; (B) NTD (pulse); (C) PM; (D) SD without Tween 80; (E) SD (1\% Tween 80); (F) SD (3\% Tween 80); (G) SD (5\% Tween 80).

Table 3. Summarization of Wavenumbers of the Characteristic Peaks of NTD-Aerosil Systems Prepared with Hypulcon

\begin{tabular}{lcc}
\hline \hline \multicolumn{1}{c}{ Sample } & -NH- group & Silanol group \\
\hline NTD & 3316 & - \\
Aerosil & - & 3434.60 \\
PM & 3319.86 & 3444.24 \\
SD (1\% Tween 80) & 3316 & - \\
SD (3\% Tween 80) & - & 3443.28 \\
SD (5\% Tween 80) & - & 3430.64 \\
Aerosil (1\% Tween 80) & - & 3375.78
\end{tabular}

It is known that silanol groups are the major adsorption sites on a silica surface and that they can interact with the adsorbate through hydrogen bonds. The interaction between drug molecules and silanol groups of silica has been reported previously. ${ }^{13-16)}$ FT-IR spectra for NTD-Aerosil systems prepared using Hypulcon (Fig. 6) were obtained to determine possible intermolecular interactions between NTD and silica particles. The wave numbers of the characteristic peaks are summarized in Table 3. NTD alone (Fig. 6A) and PM (Fig. 6C) showed $\mathrm{N}-\mathrm{H}$ stretching vibrations due to the secondary amine groups (-NH-) of NTD at 3316 and $3319.8 \mathrm{~cm}^{-1}$, respectively, which were also observed for the NTD-Aerosil SD prepared with $1 \%(\mathrm{v} / \mathrm{v})$ Tween 80 solution (Fig. 6D). However, these $\mathrm{N}-\mathrm{H}$ stretching vibrations disappeared and the characteristic absorption peaks due to the silanol groups made shifts in the IR spectra of NTD-Aerosil SDs prepared with 3\% (v/v) and 5\% (v/v) Tween 80 solutions (Figs. 6E, F). To confirm that the shifts of silanol groups were due to the interactions between NTD and Aerosil or between Tween 80 and Aerosil, the FT-IR spectrum of Aerosil with $1 \%(\mathrm{v} / \mathrm{v})$ Tween 80 solution dried with Hypulcon was obtained as a control (Fig. 6G). It was found that silanol groups made red shifts in the case of PM and all the SDs compared with Aerosil with $1 \%$ (v/v) Tween 80 solution dried with Hypulcon, as shown in Table 3. It was considered that these shifts that were not affected by the effect of Tween 80 on the shifts of silanol groups were related to the interaction with $-\mathrm{NH}-$ groups. All the above findings suggest that the hydrogen bonds between the secondary amine groups of NTD and

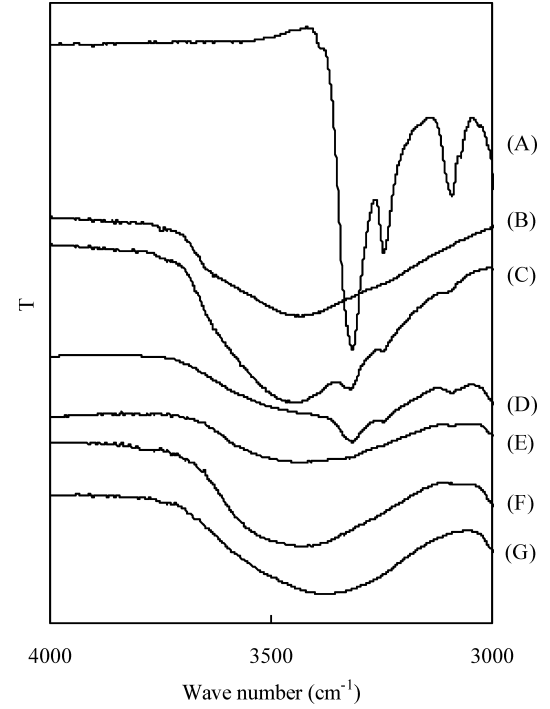

Fig. 6. FT-IR Spectra of NTD-Aerosil Systems Prepared with Hypulcon (A) original NTD; (B) Aerosil; (C) PM; (D) SD (1\% Tween 80); (E) SD (3\% Tween 80); (F) SD (5\% Tween 80$)$; (G) Aerosil (1\% Tween 80 ).

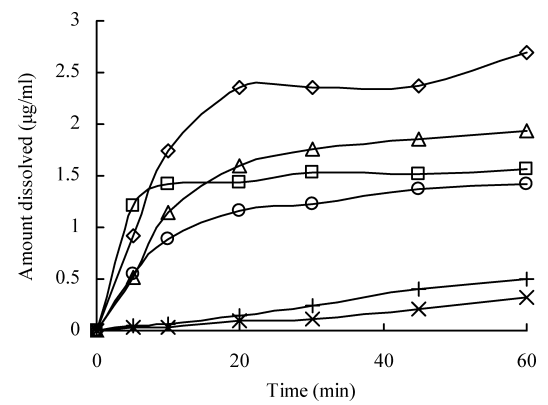

Fig. 7. Dissolution Profiles of NTD from SD Particles Prepared without Tween 80 with Hypulcon

$\times$, original NTD; +, NTD (pulse);, NTD : PVA $=1: 5 ; \square, \mathrm{NTD}: \mathrm{PVP}=1: 5 ; \triangle$, NTD : Sylysia $=1: 5 ; \diamond$, NTD $:$ Aerosil $=1: 5$

silanol groups of silica formed during the formation of SDs using the pulse combustion dryer system.

Dissolution Test Dissolution profiles of NTD from SD particles prepared without Tween 80 with Hypulcon are presented in Fig. 7. For pure NTD crystals, at the end of the dissolution test $(60 \mathrm{~min})$ the concentration of NTD was only $0.32 \mu \mathrm{g} / \mathrm{ml}$, indicating very poor dissolution of the drug. The dissolution rate of NTD dried with the pulse combustion dryer system was slightly higher than that of the original NTD. This might be due to the decrease in particle size after the use of Hypulcon. The dissolution of NTD-Aerosil SDs was significantly higher than that of SDs prepared with Sylysia, PVP, and PVA. The rank order of the drug concentration at $60 \mathrm{~min}$ for SDs was Aerosil $(2.69 \mu \mathrm{g} / \mathrm{ml})>$ Sylysia $(1.93 \mu \mathrm{g} / \mathrm{ml})>$ PVP $\quad(1.57 \mu \mathrm{g} / \mathrm{ml})>$ PVA $\quad(1.41 \mu \mathrm{g} / \mathrm{ml})$, suggesting that the dissolution of NTD was most improved by Aerosil. Aerosil is non porous nano-sized silica particles with a large specific surface area and its physicochemical properties have been described. ${ }^{7)}$ It is likely that the good hydrophilicity and dispersibility of Aerosil improved the wettability and drug dissolution from the SD.

To improve the dissolution further, SD particles were prepared with Tween 80 solutions with Hypulcon using Aerosil and Sylysia as carriers. The dissolution profiles of NTD 


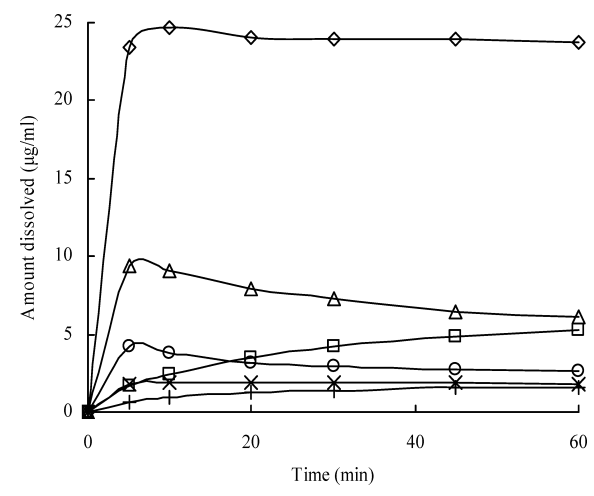

Fig. 8. Dissolution Profiles of NTD from SD Particles Prepared with Tween 80 Using Hypulcon

+ , PM (NTD : Aerosil=1:5); $\times$, NTD : Aerosil=1:5 (1\% Tween 80); $\triangle$, NTD : Aerosil =1:5 (3\% Tween 80); $\diamond$, NTD : Aerosil=1:5 (5\% Tween 80); $\bigcirc$, NTD : Sylysia $=1: 5(5 \%$ Tween 80$) ; \square$, PM (NTD : Aerosil=1:5), the dissolution medium: $0.05 \%$ Tween 80 solution.

are presented in Fig. 8. The dissolution of NTD from NTD-Aerosil SDs prepared with 3\% (v/v) and 5\% (v/v) Tween 80 solutions was significantly greater than that from pure NTD, PM, and NTD-Aerosil SD without Tween 80. The highest supersaturation level of the drug was observed for NTD-Aerosil SD prepared with $5 \%(\mathrm{v} / \mathrm{v})$ Tween 80 solution and at the end of the dissolution test $(60 \mathrm{~min})$, the concentration of NTD was $23.73 \mu \mathrm{g} / \mathrm{ml}$, about 73 -fold that of the original NTD crystals. This could be ascribed to the amorphization of the drug and the solubilization of Tween 80 . However, in the case of NTD-Sylysia SD prepared with Tween 80 solution of the same concentration $[5 \%(\mathrm{v} / \mathrm{v})]$, the dissolution of NTD was significantly lower than that of NTD-Aerosil SD prepared with 5\% (v/v) Tween 80 solution. It was confirmed that when used with $5 \%(\mathrm{v} / \mathrm{v})$ Tween 80 solution, non porous Aerosil has a greater ability to improve the dissolution of NTD than the porous Sylysia. Previously, we reported the physicochemical properties of these two types of silica and found that Aerosil had better dispersibility and wettability when used to improve the dissolution of NTD. ${ }^{7)}$ That result implied that not only the solubilization of Tween 80 but also the good dispersibility of Aerosil contributed to the improved drug dissolution from the SD.

As a control, the dissolution medium was changed from $900 \mathrm{ml}$ of water to $900 \mathrm{ml}$ of $0.05 \%$ (v/v) Tween 80 solution. The amount of Tween 80 in the dissolution medium was equivalent to that in NTD-Aerosil SD prepared with $5 \%$ (v/v) Tween 80 solution containing $40 \mathrm{mg}$ of NTD. It was found that the dissolution rate of NTD from NTD-Aerosil PM with $0.05 \%(\mathrm{v} / \mathrm{v})$ Tween 80 solution as the dissolution medium was significantly lower than that of NTD-Aerosil SD prepared with $5 \%(\mathrm{v} / \mathrm{v})$ Tween 80 with Hypulcon. This result confirmed that even if the dissolution medium contains the same amount of Tween 80, samples prepared with Hypulcon show significantly greater dissolution than PMs, indicating that the shock waves produced by the pulse engine played an indispensable role in promoting the dispersion of NTD in the carriers and the amorphization of the drug.

Dissolution Profiles of NTD from SD particles prepared with the spray dryer are presented in Fig. 9 and were compared with those prepared with Hypulcon. The dissolution from the NTD-Aerosil SD prepared using the spray dryer

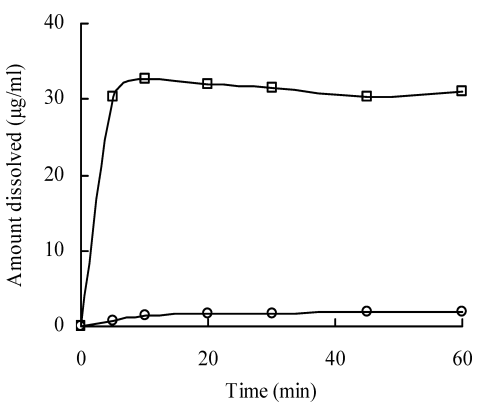

Fig. 9. Dissolution Profiles of NTD from SD Particles Prepared with the Spray Dryer

O, NTD : Aerosil $=1: 5 ; \square$, NTD : Aerosil $=1: 5$ (5\% Tween 80$)$.

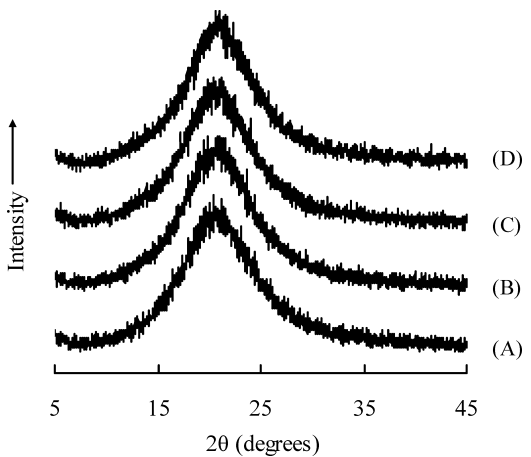

Fig. 10. Stability of Amorphous NTD in SD Particles Prepared with Hypulcon and the Spray Dryer (Stored at $75 \% \mathrm{RH}$ and $40{ }^{\circ} \mathrm{C}$ )

Hypulcon, (A) day 0; (B) 1 month; spray dryer, (C) day 0; (D) 1 month.

was lower than from the SD prepared with Hypulcon. In contrast, the NTD-Aerosil SD (5\% Tween 80) prepared with the spray dryer showed a higher rate of dissolution than that prepared with Hypulcon.

Moreover, the particles of pure NTD and PMs were found to be floating on the surface of the dissolution medium during the dissolution experiment. Conversely, the NTD-Aerosil SDs were well dispersed as soon as they were added to the dissolution medium. This result implies that the hydrophilic properties of the Aerosil contribute to the improved wettability and improved drug dissolution from the SD. Accordingly, both the amorphization of the drug and the good wettability and dispersibility of SDs might account for the rapid dissolution of SDs.

Stability Test Figure 10 shows the stability of amorphous NTD in the SD particles stored at $75 \% \mathrm{RH}$ and $40{ }^{\circ} \mathrm{C}$. It was found that the amorphous state of NTD in the NTD-Aerosil SD (5\% Tween 80) prepared with Hypulcon and the spray dryer was well maintained under moist conditions within 1 month. This may be attributed to the hydrogen bonds between the secondary amine groups of NTD and the silanol groups of Aerosil in the SDs which inhibited the crystallization of the drug from the amorphous state.

Confirmation of Particle Morphology SEM photographs of the NTD-Aerosil Systems prepared with the pulse combustion dryer system and spray dryer are shown in Figs. 11 and 12, respectively. Pure NTD was observed to be bars of crystal (Fig. 11A). NTD dried with Hypulcon was observed to remain crystals, only smaller than the original NTD (Fig. 11B). In the NTD-Aerosil PM (Fig. 11C), residual crystals of NTD were observed between the silica particles. 
For all the SDs (Figs. 11D-G), spherical particles were formed and well dispersed without any NTD crystals observed, and the particle size tended to decrease with the in-

(A)

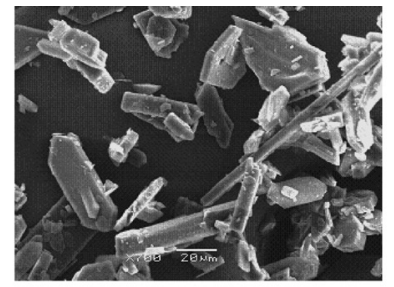

(C)

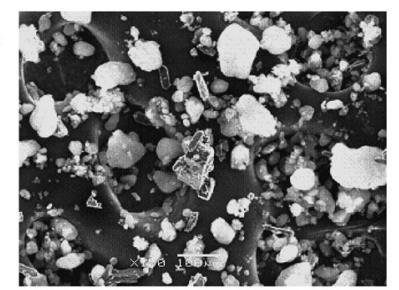

(E)

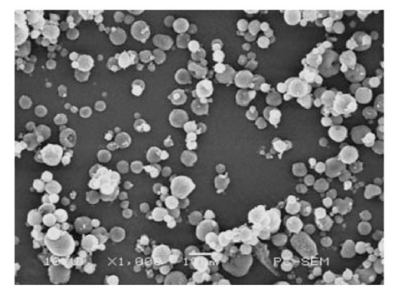

(G)

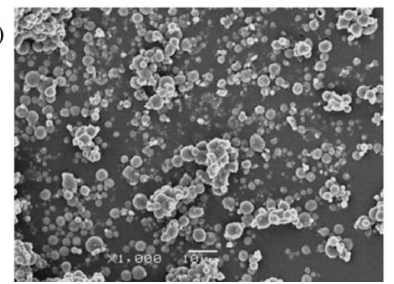

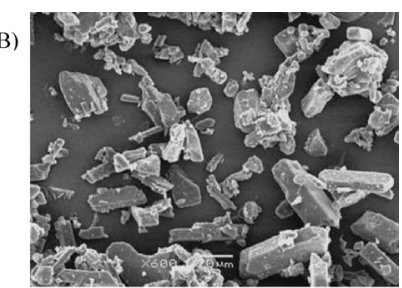
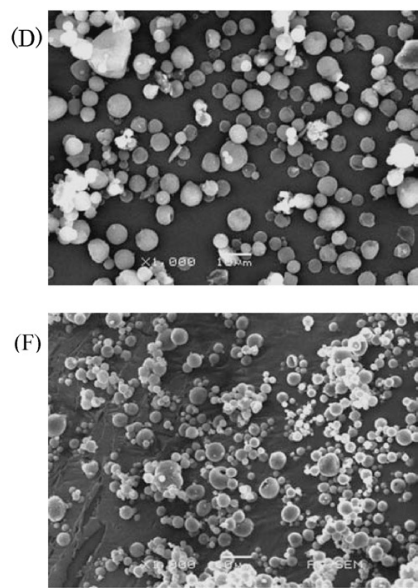

Fig. 11. Scanning Electron Micrographs of NTD-Aerosil Systems Prepared with Hypulcon

(A) Original NTD; (B) NTD (pulse); (C) PM; (D) SD without Tween 80; (E) SD (1\% Tween 80); (F) SD (3\% Tween 80); (G) SD (5\% Tween 80 ).

(A)

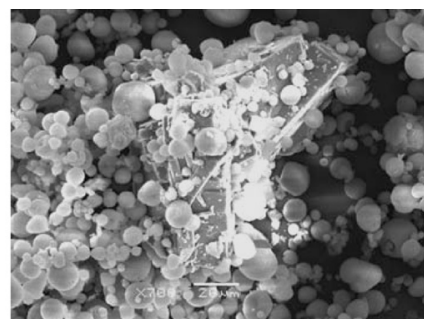

crease in the amount of Tween 80.

The SDs (Figs. 12A, B) prepared with the spray dryer were observed to be spherical particles, but formed agglomerates. Moreover, in the case of the NTD-Aerosil SD, residual crystals of NTD were observed between the spherical particles. It was suggested that the shock waves produced by Hypulcon improved the dispersion of NTD and prevented the samples from agglomerating.

Particle Size and Surface Tension The particle size of NTD-Aerosil systems was measured (Table 4). Among the samples dried with Hypulcon, the SD particles prepared without Tween 80 were larger than those prepared with Tween 80. It was found that particle size tended to decrease and particle size distribution became tighter with the increase in the concentration of Tween 80 solution.

The surface tension of Tween 80 solutions was also measured (Table 5). The surface tension decreased when the concentration of Tween 80 increased. Hence, the decrease in particle size maybe attributed to the decrease in the surface tension of Tween 80 solutions.

Among samples prepared with the spray dryer, particles were obviously larger and particle size distribution was wider. The $\mathrm{D}_{50}$ of SD particles prepared without Tween 80 was almost the same as that of SD prepared with 5\% Tween 80 solution.

\section{Conclusions}

In the present study, SD particles using Aerosil and Tween 80 as carriers were prepared with a pulse combustion dryer system, Hypulcon, under a low drying temperature $\left(60^{\circ} \mathrm{C}\right)$ without using any organic solvent. NTD in the suspensions was transformed to an amorphous state by the actions of shock waves and ultrasonic waves produced by Hypulcon. The good hydrophilicity and dispersibility of Aerosil and the solubilization and low surface tension of Tween 80 also played important roles in the amorphization of NTD and improved the dissolution of SDs. Samples treated with Hypulcon showed improved properties over those prepared with a

(B)

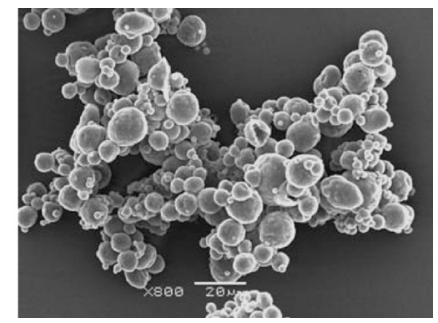

Fig. 12. Scanning Electron Micrographs of NTD-Aerosil Systems Prepared with the Spray Dryer

(A) SD without Tween 80; (B) SD (5\% Tween 80).

Table 4. Particle Size of Solid Dispersion Particles

\begin{tabular}{|c|c|c|c|c|}
\hline \multirow{2}{*}{ Method } & \multirow{2}{*}{ Sample } & \multicolumn{3}{|c|}{ Particle size $(\mu \mathrm{m})$} \\
\hline & & $\mathrm{D}_{10}$ & $\mathrm{D}_{50}$ & $\mathrm{D}_{90}$ \\
\hline \multirow[t]{4}{*}{ Hypulcon } & NTD $:$ Aerosil $=1: 5$ & 3.20 & 4.75 & 6.70 \\
\hline & NTD : Aerosil=1:5(1\% Tween 80$)$ & 2.30 & 3.75 & 5.90 \\
\hline & NTD : Aerosil=1:5 (3\% Tween 80$)$ & 1.80 & 3.10 & 4.60 \\
\hline & NTD : Aerosil=1:5 (5\% Tween 80$)$ & 1.75 & 2.50 & 4.10 \\
\hline \multirow[t]{2}{*}{ Spray drying } & NTD : Aerosil $=1: 5$ & 3.20 & 6.20 & 11.60 \\
\hline & NTD : Aerosil=1:5 (5\% Tween 80$)$ & 2.60 & 6.00 & 12.80 \\
\hline
\end{tabular}


Table 5. Surface Tension of Tween 80 Solutions at $30^{\circ} \mathrm{C}$

\begin{tabular}{cc}
\hline \hline Solution & Surface tension $(\mathrm{dyn} / \mathrm{cm})$ \\
\hline 1\% Tween 80 & 42.00 \\
3\% Tween 80 & 40.13 \\
$5 \%$ Tween 80 & 37.84 \\
\hline
\end{tabular}

conventional spray dryer, such as smaller particle size, tighter particle size distribution, and no agglomeration. It was also observed that the drug supersaturation concentration was maintained continuously during the dissolution test of the NTD-Aerosil SD prepared with 5\% Tween 80 solution and Hypulcon. The problem of the decrease in the drug supersaturation concentration during the dissolution test was resolved. ${ }^{7)}$ It was confirmed that the pulse combustion dryer system can be used to prepare SD particles with improved properties without using any organic solvent.

\section{References}

1) Sekiguchi K., Obi N., Chem. Pharm. Bull., 9, 866-872 (1961).

2) Chiou W. L., Riegelman S., J. Pharm. Sci., 59, 937-942 (1970).

3) Chiou W. L., Riegelman S., J. Pharm. Sci., 60, 1281-1302 (1971).

4) Ford J. L., Stewart A. F., Rubinstein M. K., J. Pharm. Pharmacol., 31
$726-729$ (1979).

5) Wang L., Cui F., Hayase T., Sunada H., Chem. Pharm. Bull., 53, $1240-1245$ (2005).

6) Wang L., Sunada H., Preparation and evaluation of solid dispersion for nitrendipine-Aerosil system using melt-mixing method, Symposia of the 125th Annual Meeting of the Pharmaceutical Society of Japan, 2005.

7) Wang L., Cui F., Sunada H., Chem. Pharm. Bull., 54, 37-43 (2006).

8) Instruction Book of Hypulcon (Mini Labo Test), Pultech, Apr. 2006.

9) Weuts I., Kempen D., Verreck G., Deorte A., Heymans K., Peetens J., Brewster M., Vandan Mooter G., Eur. J. Pharm. Biopharm., 59, 119126 (2005).

10) Paradkar A., Ambike A. A., Jadhav B. K., Jadhav B. K., Mahadik K. R., Int. J. Pharm., 271, 281-286 (2004).

11) Kudra T., Mujumdra A. S., "Advanced Drying Technologies," Marcel Dekker Inc., New York, 2001.

12) Kudra T., Mujumdra A. S., "Hand Book of Industrial Drying," ed. by Mujumdra A. S., Marcel Dekker Inc., New York, 1995, pp. 10871149 .

13) Takeuchi H., Nagira S., Yamamoto H., Kawashima Y., Int. J. Pharm., 293, 155-164 (2005).

14) Watanabe T., Wakiyama N., Usui F., Ikeda M., Isobe T., Senna M., Int J. Pharm., 226, 81-91 (2001).

15) Watanabe T., Ohno I., Wakiyama N., Kusai A., Senna M., STP Pharm. Sci., 12, 363-367 (2002).

16) Watanabe T., Hasegawa S., Wakiyama N., Kusai A., Senna M., Int. J. Pharm., 250, 283-286 (2003). 